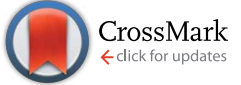

Cite this: J. Mater. Chem. A, 2015, 3, 3839

Received 15th November 2014 Accepted 22nd December 2014

DOI: $10.1039 / c 4 t a 06222 a$

www.rsc.org/MaterialsA

\title{
A porosity difference based selective dissolution strategy to prepare shape-tailored hollow mesoporous silica nanoparticles $\uparrow$
}

\begin{abstract}
Adem Yildirim $^{\star a b}$ and Mehmet Bayindir*abc
This article reports a general method to prepare hollow mesoporous silica nanoparticles with tailored morphology. The method is based on selective dissolution of porous cores of solid silica shell/porous silica core nanoparticles under mild conditions without the need for corrosive or toxic etchants. First, core-shell nanospheres or nanorods are prepared in a one-pot reaction. Then, mesoporous cores of the nanoparticles are selectively dissolved by incubating them in phosphate buffered saline (PBS) at $65^{\circ} \mathrm{C}$ for one day. Surprisingly, shells of the resulting hollow particles contain both small and large mesopores which makes the particles very suitable for adsorption and desorption of a wide range of molecules. In addition, we proposed a mechanism for selective dissolution of porous cores of the core-shell nanoparticles.
\end{abstract}

\section{Introduction}

Hollow silica nanostructures are attracting a great deal of interest in many research areas including catalysis, controlled drug delivery, bioimaging, optical coatings and absorption due to their unique properties such as high surface area and pore volume, ease of surface functionalization, chemical stability and low toxicity. ${ }^{1-3}$ They are often prepared using template based methods in which a variety of hard ${ }^{\mathbf{4 - 1 1}}$ (polymer or inorganic micro/nano-particles) or soft ${ }^{\mathbf{1 2 - 1 9}}$ templates (droplets, bubbles, micelles, etc.) can be applied. However, template based methods have some drawbacks which prevent their widespread applications. For instance, hard templating methods have tedious multistep and time-consuming procedures including core synthesis, surface functionalization of core, silica deposition and core etching. Soft template based methods, on the other hand, involve less synthesis steps; however products of these methods often have ill-defined shapes and are polydisperse in size due to the deformability of soft-templates. ${ }^{1}$ Recently, templateless or homogenous templating methods such as surface protected etching ${ }^{20-22}$ and selective etching ${ }^{23-28}$ have emerged to fulfil the need for simple, effective and scalable synthesis of hollow silica nanostructures. However, these

\footnotetext{
${ }^{a}$ UNAM-National Nanotechnology Research Center, Bilkent University, 06800 Ankara, Turkey.E-mail: yildirim.adem83@gmail.com; ademy@bilkent.edu.tr

${ }^{b}$ Institute of Materials Science and Nanotechnology, Bilkent University, 06800 Ankara, Turkey

'Department of Physics, Bilkent University, 06800, Ankara, Turkey.E-mail: bayindir@ nano.org.tr; Fax: +90 312266 4365; Tel: +90 3122903500

$\uparrow$ Electronic supplementary information (ESI) available: A PDF containing additional TEM images and FTIR spectra. See DOI: 10.1039/c4ta06222a
}

methods usually require corrosive and toxic etching agents (strong alkaline or HF solutions) to selectively etch cores of solid silica particles. A few recent studies reported that under mild conditions (water or slightly acidic solutions) silica nano/ microparticles can undergo spontaneous structural change from solid spheres into hollow particles. ${ }^{29-33}$ Unfortunately, in these methods special care must be taken to control the shell thickness and particle morphology since they are very dependent on the reaction conditions such as $\mathrm{pH}$, temperature, and etch time. Also, the shells of the resulting hollow particles usually do not have accessible mesopores which restrict their applications in many fields such as controlled drug release, catalysis and pollutant adsorption. In addition, the shape of the hollow particles prepared through the spontaneous structural change process is generally limited to spheres since their synthesis often started with spherical Stöber silica nanoparticles. ${ }^{34}$ Other than spheres, rods or tubes can be more desired for some applications. For example, rod shaped particles are proved to be more effective in biological applications than their spherical counterparts due to their higher cellular uptake. ${ }^{35}$ Overall, facile and scalable preparation of hollow silica nanostructures with tailored shape and porosity is still a great challenge.

In this article, we report a porosity difference based selective dissolution strategy to prepare hollow silica nanospheres and nanorods with mesopores on their shells using a mild etching agent; phosphate buffered saline (PBS) (Fig. 1). The method is based on the selective dissolution of mesoporous silica cores of solid silica shell/mesoporous silica core nanoparticles, which gives a good control over the particle size, shell thickness, porosity and particle shape (sphere or rod). The method involves two steps; (i) synthesis of core-shell nanospheres or 
nanorods in a one-pot reaction and (ii) selective etching of mesoporous cores in PBS. The particle morphology can be controlled using Rose Bengal (RB) dye. Rod-shaped mesoporous silica nanoparticles (MSNs) with controllable aspect ratio can be prepared in the presence of $\mathrm{RB}$; in the absence of $\mathrm{RB}$ particles are spherical. Solid silica shells can be coated over these particles by addition of the excess silica precursor during the synthesis. To our surprise, during the hollowing process, mesopores developed on the solid silica shells which provide accessibility to the hollow interior of the particles.

\section{Experimental section}

\section{Materials}

Cetyltriammoniumbromide (CTAB), F127 Pluronic polymer, and Rose Bengal were purchased from Sigma Aldrich (USA). Tetraethyl orthosilicate (TEOS) was purchased from Merck (Germany). All chemicals were used as purchased.

\section{Preparation of mesoporous silica nanospheres and nanorods}

Nanospheres were prepared by modifying previous reports. ${ }^{36-38}$ Briefly, $200 \mathrm{mg}$ of CTAB was dissolved in $95 \mathrm{~mL}$ of water and 1 $\mathrm{mL}$ of $\mathrm{F} 127$ solution ( $5 \mathrm{mg} \mathrm{mL} \mathrm{m}^{-1}$ in water) and $0.7 \mathrm{~mL}$ of $2 \mathrm{M}$ $\mathrm{NaOH}$ were added to this solution. Then the reaction mixture was heated to $80^{\circ} \mathrm{C}$ while stirring vigorously (600 rpm). After the temperature was fixed at $80{ }^{\circ} \mathrm{C}, 1.2 \mathrm{~mL}$ of TEOS was rapidly added and the reaction mixture was further stirred for $2 \mathrm{~h}$. Finally, reaction solution was cooled down to room temperature and particles were collected by centrifugation at $9000 \mathrm{rpm}$ for 20 min and washed with water twice. To synthesize nanorods, we added an appropriate amount of RB, (for example $10 \mathrm{mg}$ for the particles with an aspect ratio of 1.9) which is dissolved in $2 \mathrm{~mL}$ of water, to the reaction mixture after addition of TEOS. Other parameters were the same as the nanosphere synthesis.

\section{Preparation of core-shell nanoparticles}

To prepare core-shell particles we added an appropriate amount of additional TEOS ( 0.5 to $3 \mathrm{~mL}$ ) after $1 \mathrm{~h}$ of first TEOS addition and the reaction mixture was further stirred for $1 \mathrm{~h}$. For example, to prepare the nanoparticles with a shell thickness of $8 \mathrm{~nm}$ we added $1 \mathrm{~mL}$ of TEOS in the second step. Other parameters were the same as the nanosphere or nanorod synthesis.

\section{Preparation of hollow nanoparticles}

Cores of the core-shell particles were dissolved by incubating the particles in PBS (10 mM, pH 7.4) at $65{ }^{\circ} \mathrm{C}$ for 1 day under gentle stirring. The particle concentration in PBS solution was $0.5 \mathrm{mg} \mathrm{mL} \mathrm{m}^{-1}$ for nanospheres and $1 \mathrm{mg} \mathrm{mL} \mathrm{m}^{-1}$ for nanorods. Particles were collected by centrifugation at $9000 \mathrm{rpm}$ for 20 min and washed with water twice.

\section{Surfactant removal}

In the final step, nanoparticles (nanospheres, nanorods, coreshell particles or hollow particles) were calcined at $550{ }^{\circ} \mathrm{C}$ for 5 hours (ramp rate was $5{ }^{\circ} \mathrm{C} \mathrm{min}^{-1}$ ) to remove the CTAB surfactant and RB molecules.

\section{Characterization}

Transmission electron microscopy (TEM) images were obtained using a Tecnai G2 F30 (FEI) microscope. The scanning electron microscopy (SEM) images of particles were obtained using an ESEM; Quanta 200F (FEI). FTIR spectra of nanospheres were obtained by using a Fourier transform infrared spectrometer (FTIR, Vertex 70, Bruker). Surface area, pore volume and pore size distribution of the particles were determined using an iQ-C (Quantachrome). X-ray powder diffraction (XRD) spectra of the particles were recorded using an X'Pert Pro (Panalytical).

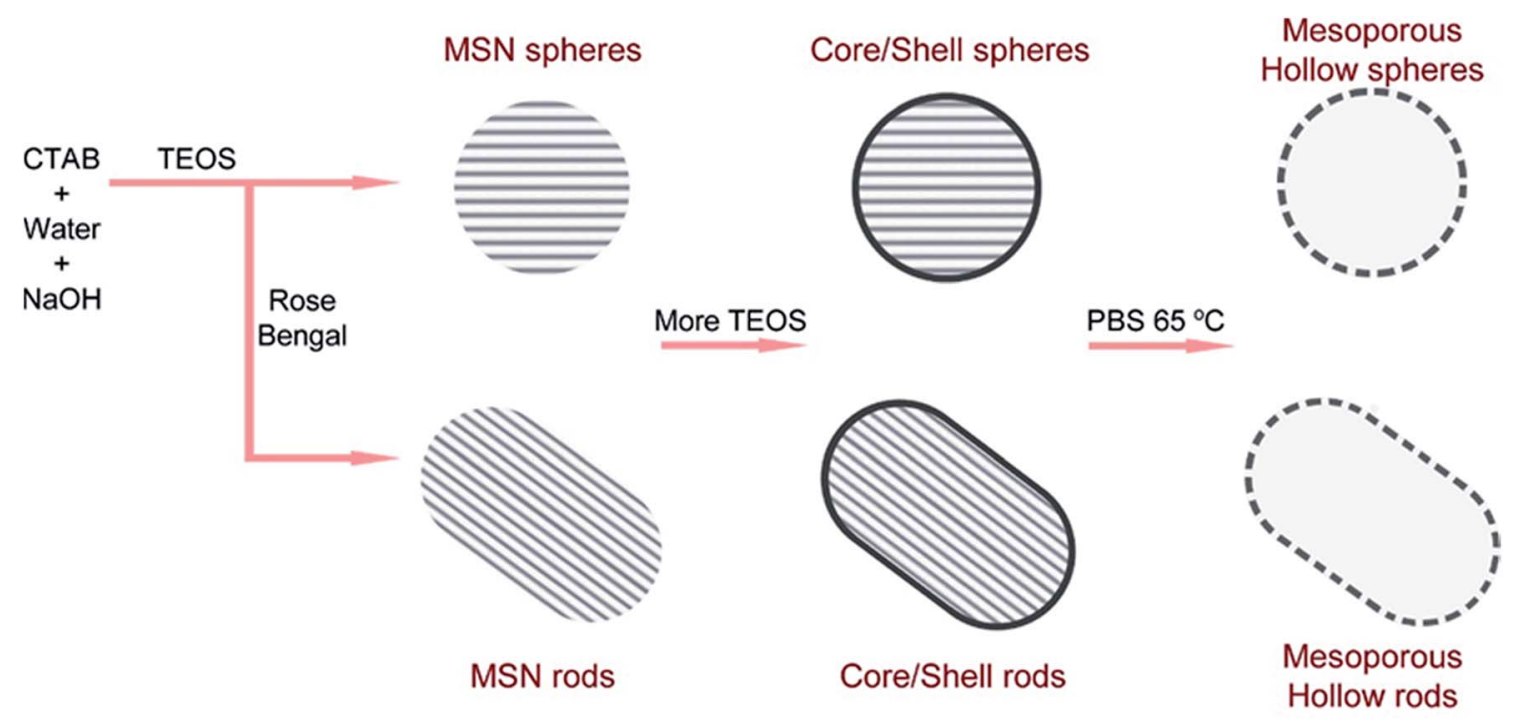

Fig. 1 Schematic representation of synthesis of hollow nanospheres and nanorods. In the presence of RB dye rod-shaped particles are formed. Addition of excess TEOS during the reaction produces a uniform silica shell around particles. PBS selectively dissolves the cores and creates mesopores on the shells of the particles. 


\section{Results and discussion}

\section{Effect of RB on morphology of MSNs}

Fig. 2 shows the effect of RB on the morphology of MSNs. In the absence of RB, particles are almost spherical (Fig. 2a and d) which is typical for MCM-41 type MSNs. ${ }^{36}$ The average particle size of spherical MSNs was calculated to be around $120 \mathrm{~nm}$ from the TEM images. Interestingly, in the presence of $5 \mathrm{mg}$ of $\mathrm{RB}$ (Fig. 2b and e) rod-shaped MSNs (aspect ratio is $\sim 1.5$ ) were obtained. Doubling the RB amount $(10 \mathrm{mg})$ increases the aspect ratio of particles to $\sim 1.9$ (Fig. $2 \mathrm{c}$ and f). The average particle width and length for rod-shaped MSNs prepared using $10 \mathrm{mg}$ of $\mathrm{RB}$ were determined to be around $75 \mathrm{~nm}$ and $145 \mathrm{~nm}$, respectively from TEM images. Further increasing the RB amount (20 $\mathrm{mg}$ ), however, results in a mixture of polydisperse spheres and very long helical rods with aspects ratios even larger than 10 (see ESI, Fig. S1†).

It is known that the morphology of the mesoporous silica materials is highly dependent on the synthesis conditions. Even a slight change in the reaction parameters such as temperature and $\mathrm{pH}$ may significantly alter the size, shape and pore structure of the MSNs by affecting the nucleation, growth and dissolution rates of silica and the thermodynamics of
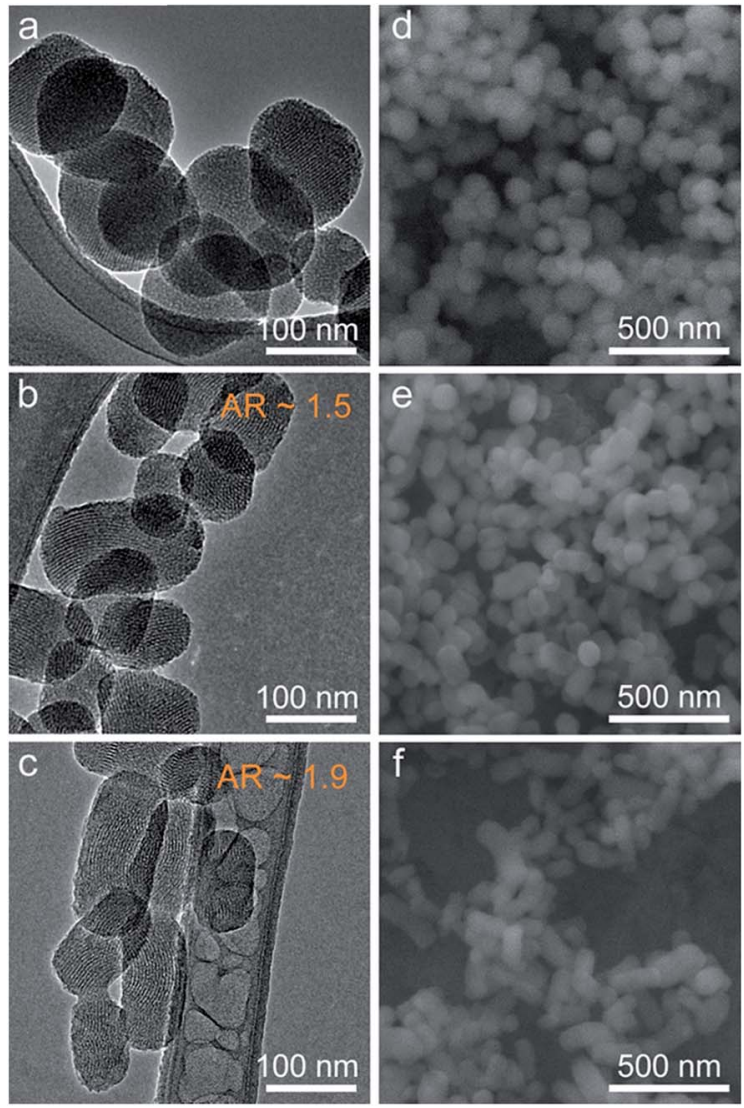

Fig. 2 TEM (left panel) and SEM (right panel) of MSNs prepared using different amounts of RB. (a and d) No RB, (b and e) $5 \mathrm{mg}$ of RB and (c and f) $10 \mathrm{mg}$ of RB. In the presence of RB, rod-shaped MSNs are formed and with the increasing RB amount longer rods can be obtained. surfactant-silica assembly. ${ }^{39}$ For instance, Mou et al. ${ }^{40}$ demonstrated the significant impact of the $\mathrm{pH}$ on the size of MSNs; decreasing the $\mathrm{pH}$ from 11.38 to 10.86 reduced the particle size almost 6 fold (170 to $30 \mathrm{~nm}$ ). Also, Yang et al. ${ }^{41}$ reported that addition of acetate esters resulted in a rapid decrease in the $\mathrm{pH}$ of the reaction mixture and changed the shape of the particles from spheres to helical rods by changing nucleation and growth rates of silica and interactions between silicates and surfactant molecules. Similarly, we hypothesized that dissolution of the RB molecules may change the $\mathrm{pH}$ of the reaction solution and this may be the reason for the rod-shape MSN formation in the presence of RB. Accordingly, we measured the $\mathrm{pH}$ of the reaction solutions before and after addition of different amounts of RB. However, we did not observe any significant change in the $\mathrm{pH}$ of the reaction mixture $(\sim 11.75)$ even after addition of $20 \mathrm{mg}$ of RB. Apparently, a pH drop based shape transformation mechanism is not applicable for our case. On the other hand, He et $a l .{ }^{42}$ reported that negatively charged ibuprofen molecules incorporate into the surfactant micelles and affect the pore morphology of the particles. Although, RB is also negatively charged, TEM images did not indicate any difference in the pore structure of the MSNs prepared in the presence or absence of RB. Therefore, incorporation of RB molecules into the surfactant micelles did not change the mesostructure of surfactant micelle assembly at least in the studied concentration region. Based on these results, it is believed that, the shape transformation in the presence of $\mathrm{RB}$ is due to the effect of negatively charged RB molecules on the assembly between silica and surfactant micelles. The negatively charged RB may electrostatically assemble with cationic surfactant micelles and accordingly can change the shape of the silica-surfactant assembly.

\section{Synthesis of core-shell MSNs}

Mesoporous core/solid silica shell particles are prepared in a one-pot reaction. Following the polymerization of the tetraethyl orthosilicate (TEOS) monomer around CTAB micelles to form initial MSNs, excess TEOS monomers were added to prepare solid shells around the particles. Fig. 3 shows the TEM images of core-shell nanospheres and nanorods which were prepared using different amounts of TEOS in the second addition. Uniform solid silica shells around the particles reflect clear contrast difference from the porous cores. Fig. $3 \mathrm{a}$ and b show the nanospheres prepared using 1 and $3 \mathrm{~mL}$ of additional TEOS which have 8 and $12 \mathrm{~nm}$ thick very uniform shells, respectively. Similarly, an $8 \mathrm{~nm}$ thick uniform silica shell was observed around the nanorods (Fig. 3c) when $1 \mathrm{~mL}$ of TEOS was used in the second step showing that the shell thickness is independent of the particle morphology. Fig. 3d demonstrates the effect of second TEOS addition amount on the silica shell thickness of nanospheres. We observed that between 0.5 and $2 \mathrm{~mL}$ of TEOS addition, the shell thickness gradually increased to $12 \mathrm{~nm}$ from $5 \mathrm{~nm}$. A further increase in the TEOS amount $(3 \mathrm{~mL})$ did not change the shell thickness significantly indicating that shell growth is self-limiting. 

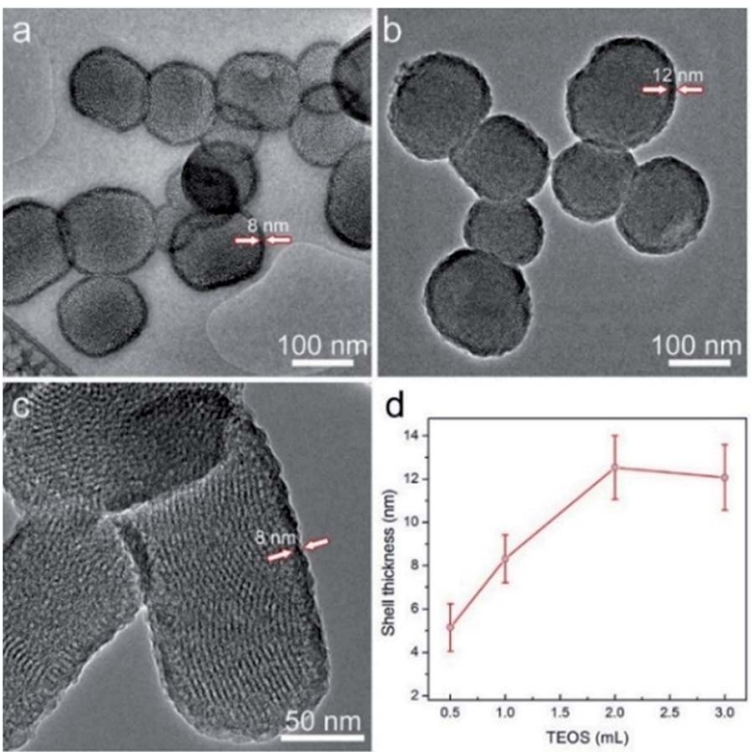

Fig. 3 Morphology and shell thickness of core-shell MSNs. (a-c) TEM images of spherical MSNs prepared using $1 \mathrm{~mL}$ of additional TEOS (a), spherical MSNs prepared using $3 \mathrm{~mL}$ of additional TEOS (b), rod-shaped MSNs prepared using $1 \mathrm{~mL}$ of additional TEOS (c). Arrows indicate the shells of the particles. (d) Shell thickness of spherical particles is determined by the additional TEOS amount.

\section{Selective dissolution of porous cores in PBS}

Porous cores of the core-shell particles were selectively dissolved by incubating the particles in PBS at $65{ }^{\circ} \mathrm{C}$ for one day (Fig. 4). After the hollowing process, the particles were calcined at $550{ }^{\circ} \mathrm{C}$ to remove the CTAB surfactant and $\mathrm{RB}$ molecules. It should be noted that the particle concentration in PBS solution was $0.5 \mathrm{mg} \mathrm{mL}^{-1}$ for nanospheres and $1 \mathrm{mg} \mathrm{mL}^{-1}$ for nanorods. We observed that partially etched particles are formed at higher particle concentrations (will be discussed below) suggesting that an equilibrium is reached for silica dissolution.

Fig. 4 shows the TEM images of fabricated hollow nanospheres (prepared using $1 \mathrm{~mL}$ of additional TEOS) and nanorods (prepared using $10 \mathrm{mg}$ of RB and $1 \mathrm{~mL}$ of additional TEOS). There is a clear contrast difference between interior and exterior parts of the particles indicating core dissolution after PBS incubation. The efficiency of the hollowing process is almost $100 \%$ (see low magnification TEM image of the hollow nanospheres in the ESI, Fig. S2†). Importantly, the shell thickness remained almost intact after the hollowing process; around 8-9 $\mathrm{nm}$, for both nanospheres and nanorods which suggests that only cores of the particles dissolved.

To investigate the selective core dissolution process, FTIR spectra of core particles (MSN), core-shell particles (MSN-ts) and hollow particles (h-MSN) were collected, after the surfactant removal step (see ESI, Fig. S3†). The broad band around 1000$1250 \mathrm{~cm}^{-1}$ is composed of several longitudinal-optical (LO) and transverse-optical (TO) modes of the $\mathrm{SiO}_{2}$ network and peaks of some residual groups bonded to this network. ${ }^{43}$ The position and shape of this broad band may provide important information about the cross-linking degree of the silica network. ${ }^{\mathbf{4 3 , 4 4}}$
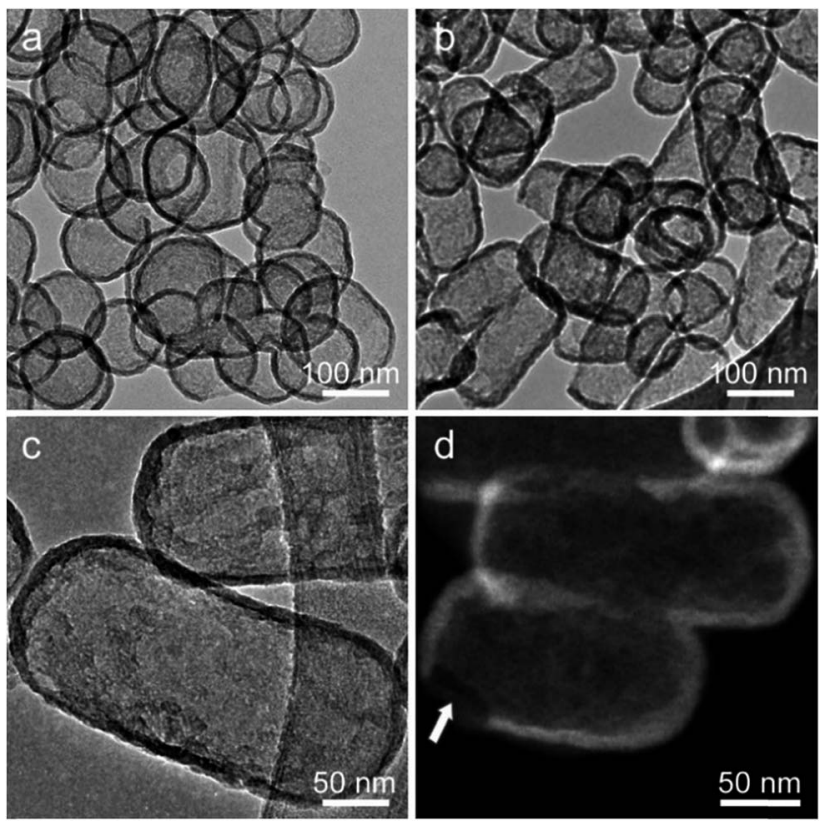

Fig. 4 TEM images of hollow nanoparticles. (a) Hollow nanospheres prepared using core-shell particles with an $8 \mathrm{~nm}$ shell thickness, (b) hollow nanorods prepared using core-shell particles with an $8 \mathrm{~nm}$ shell thickness. The shell thickness of hollow particles remained the same after the hollowing process, indicating that cores were completely dissolved. (c) Close-up TEM image of nanorods. (d) High magnification STEM image of hollow nanorods that shows the mesoporous shells of particles. Arrow indicates a crack formed on the hollow nanorod shell during core dissolution and calcination processes.

The maximum of this band (at $1082 \mathrm{~cm}^{-1}$ ) of MSN blue shifted to around $1085 \mathrm{~cm}^{-1}$ after silica shell coating (MSN-ts) and further shifted to $1090 \mathrm{~cm}^{-1}$ after the hollowing process (hMSN). A blue shift in this peak indicates more cross-linked structure with higher internal stress. ${ }^{\mathbf{4 4 , 4 5}}$ In addition, the intensity of the shoulder around $1200 \mathrm{~cm}^{-1}$ decreases with the increasing cross-linking degree of the silica network. ${ }^{43,46}$ An intense shoulder at $1235 \mathrm{~cm}^{-1}$ was observed for the MSN indicating a poorly cross-linked silica network for the core particles. After coating the MSN with a thin silica shell (MSN-ts) the intensity of the shoulder decreased and it further reduced after core removal (h-MSN). These results suggest that the shell is more cross-linked than the core. Therefore, the less cross-linked and porous core is more susceptible for dissolution and PBS selectively etches the cores of the core-shell particles.

To our surprise, shells of the particles contain mesopores after dissolution which can be clearly seen from Fig. 4c and d. The mesoporosity of the shells was further confirmed using an $\mathrm{N}_{2}$ adsorption-desorption technique (see below). The mesopores can be formed during the silica dissolution process in the presence of surfactant molecules. ${ }^{24}$ It is well known that dissolution of silica is reversible (Scheme 1) and therefore dissolved silica species can spontaneously regrow. ${ }^{29}$ For our case, in the presence of CTAB (leaking from dissolved cores of the particles) dissolved silica can regrow around $\mathrm{CTAB}$ micelles to produce the mesoporous shell. Another reason for the 
<smiles>CO[C@@H](O[Si](OC)(OC)OC)O[Si](OC)(OC)O[C@@H](O)O[Si](OC)(OC)OC</smiles>

Scheme 1 Mechanism of hydroxide ion catalyst silica dissolution and regrowth.

mesopore formation can be the residual CTAB molecules which are adsorbed to the shell silica network during the synthesis of particles. These residual CTAB molecules can contribute to the pore formation during dissolution, regrowth and calcination processes. ${ }^{24}$ Besides, we observed cracks on the some of the particles after the calcination process (Fig. 4d). Nevertheless, according to the TEM images most of the particles were crack free.

Pore structure, volume, size and specific surface area of rodshaped particles were characterized using $\mathrm{N}_{2}$ adsorptiondesorption and XRD techniques. Fig. 5a shows the $\mathrm{N}_{2}$ adsorption-desorption curves of nanorods (rMSN), core-shell nanorods (rMSN-ts), and hollow nanorods (h-rMSN). rMSN and rMSN-ts revealed a type-IV nitrogen sorption curve with a sharp capillary condensation step in the nitrogen adsorption amount at relative pressures $P / P_{0}$ between 0.2 and 0.4 indicating the presence of mesopores with narrow size distribution. ${ }^{11,30}$ On the other hand, this step almost disappeared for $\mathrm{h}$ rMSN reflecting the dissolution of an ordered mesoporous core. Corresponding pore size distributions of the particles, which were obtained from $\mathrm{N}_{2}$ adsorption-desorption curves using the DFT method, are given in Fig. 5b. rMSN and rMSN-ts revealed a similar narrow pore size distribution with mesopores; $2.9 \mathrm{~nm}$ and $3 \mathrm{~nm}$, respectively. The pore size distribution of h-rMSN, on the other hand, was wide between 2 and 6 $\mathrm{nm}$ which may be due to the random nature of the silica dissolution and regrowth process and pore widening during core dissolution and calcination processes. Also, a second wide peak was observed in the h-rMSN pore size distribution between 13 and $22 \mathrm{~nm}$ which may correspond to the cracks formed on the shell during dissolution and calcination processes.

The surface area and pore volume of the particles were calculated using BET and DFT methods, respectively. The surface area of rMSN is $1008 \mathrm{~m}^{2} \mathrm{~g}^{-1}$ and it is reduced to 862 $\mathrm{m}^{2} \mathrm{~g}^{-1}$ after coating the particles with a solid silica shell (rMSN-ts). The surface area further reduced to $598 \mathrm{~m}^{2} \mathrm{~g}^{-1}$ after core dissolution (h-rMSN) due to the loss of highly ordered and mesoporous cores. The pore volume of rMSN is 1 $\mathrm{cm}^{3} \mathrm{~g}^{-1}$ and it is again slightly reduced $\left(0.87 \mathrm{~cm}^{3} \mathrm{~g}^{-1}\right)$ after silica coating. As expected, the pore volume increases more than two fold $\left(1.94 \mathrm{~cm}^{3} \mathrm{~g}^{-1}\right)$ after etching the core of the particles.

Fig. 6 shows the XRD spectra of the rod-shaped particles. rMSN and rMSN-ts exhibited characteristic diffraction peaks, (100), (110), and (200), of the highly ordered hexagonal pore structure of MCM-41 type mesoporous materials; whereas no order was observed for h-rMSN. Based on TEM, $\mathrm{N}_{2}$ adsorption-
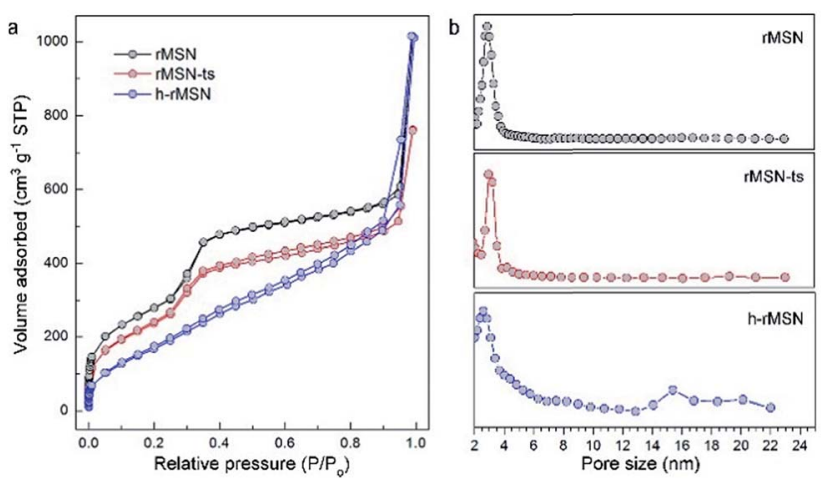

Fig. 5 (a) $\mathrm{N}_{2}$ adsorption and desorption curves of rod-shaped nanoparticles. The step in nitrogen adsorption amount at relative pressures $P / P_{0}$ between 0.2 and 0.4 disappeared for hollow particles indicating the loss of the mesoporous core. (b) Pore size distribution of particles calculated using the $\mathrm{N}_{2}$ adsorption and desorption curves. rMSN and rMSN-ts showed narrow pore size distribution, on the other hand, hrMSN have broad pore size distribution (rMSN; rod-shaped MSNs, rMSN-ts; rod-shaped core-shell MSNs, and h-rMSN; rod-shaped hollow MSNs).

desorption and XRD observations, it can be concluded that shells of hollow particles contain non-ordered mesopores with large pore size distribution.

Using the porosity difference based selective dissolution method, it is possible to prepare particles with different morphologies. For instance, Fig. 7 shows the TEM images of the partially hollowed nanospheres. These particles contain a few large pores in their cores. Also, it is still possible to observe ordered mesopores in the non-dissolved parts (Fig. 7b). To prepare the half-etched particles we increased the particle concentration to $5 \mathrm{mg} \mathrm{mL} \mathrm{m}^{-1}$ and we kept the dissolution time and temperature constant. Note that the reaction is spontaneously quenched at this particle concentration; further

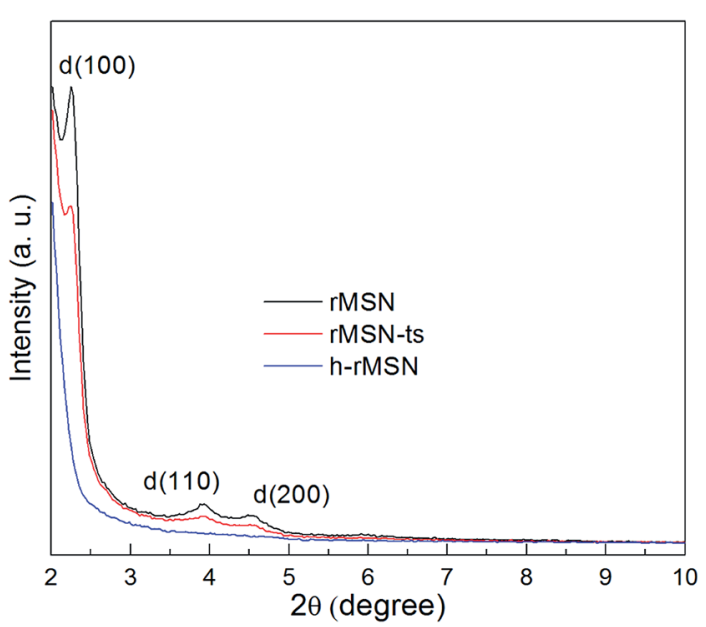

Fig. 6 XRD spectra of rod-shaped particles. rMSN and rMSN-ts, demonstrated characteristic diffraction peaks, (100), (110), and (200), of the highly ordered hexagonal pore structure of MCM-41 type mesoporous materials. On the other hand, pore order disappeared after core dissolution (h-rMSN). 

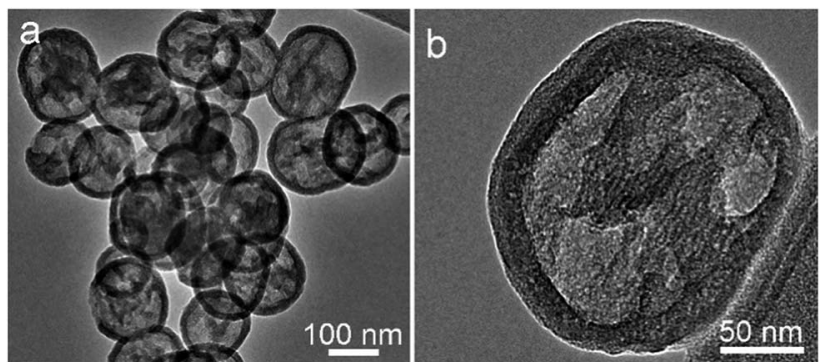

Fig. 7 TEM images of partially hollowed spherical particles (a) low magnification and (b) high magnification. Partially hollowed particles contain a few large pores in their cores and it is still possible to observe ordered mesopores in the non-dissolved parts.

increasing the reaction time does not change the particle morphology. Therefore, it is possible to partially dissolve the cores of core-shell particles by simply changing the particle concentration in the PBS solution.

\section{Possible mechanism of selective dissolution process}

In order to investigate the selective dissolution mechanism, we collected samples at different time intervals during the incubation of rMSN-ts in PBS at $65^{\circ} \mathrm{C}$ (particle concentration is $1 \mathrm{mg}$ $\mathrm{mL}^{-1}$ ). However, we noticed that at this temperature core dissolution is very fast; cores of the particles were completely etched after $30 \mathrm{~min}$, which prevent us from the observation of dissolution steps. To slow down the reaction we decreased the dissolution temperature to room temperature and increased the particle concentration to $5 \mathrm{mg} \mathrm{mL}^{-1}$. As expected, the core dissolution is very slow under these conditions. At the beginning of the dissolution process (after 1 day), many small pores around $10 \mathrm{~nm}$ were observed (Fig. 8a). After 3 days (Fig. 8b) pore sizes increased and further increased after 1 week (Fig. 8c). This indicates that core etch starts from multiple points, and these sites became wider with time and then interconnected with each other. At the end of the process all of the pores were interconnected to form the hollow core (Fig. 8d).

To explore the role of PBS in the dissolution process, we replaced PBS with water and incubated rMSN-ts at $65^{\circ} \mathrm{C}$ for one day. We observed that in water some of the cores were not fully dissolved (see ESI, Fig. S4 $\dagger$ ) indicating that dissolution in water is slower than in a highly salted PBS environment. In fact, it is well known that silica dissolution is faster in salted solutions ${ }^{33,47}$ which is in accordance with our observations. Nevertheless, cores of particles can be dissolved in water by decreasing the particle concentration.

We also investigated the effect of calcination on the selective dissolution process. In this case, first we calcined the particles at $550{ }^{\circ} \mathrm{C}$ for $5 \mathrm{~h}$ in order to strengthen the silica network, then we incubated the particles in PBS at $65^{\circ} \mathrm{C}$ for one day. One can expect no or partially dissolved cores for this case due to the improved condensation degree of silica after high temperature treatment. ${ }^{48}$ In contrast, we observed that cores of the particles dissolved similar to the non-calcined particles (see ESI, Fig. S5 $\dagger$ ). However, the shell thickness of the pre-calcined particles are almost two times thicker $(14 \mathrm{~nm})$ than non-precalcined particles.
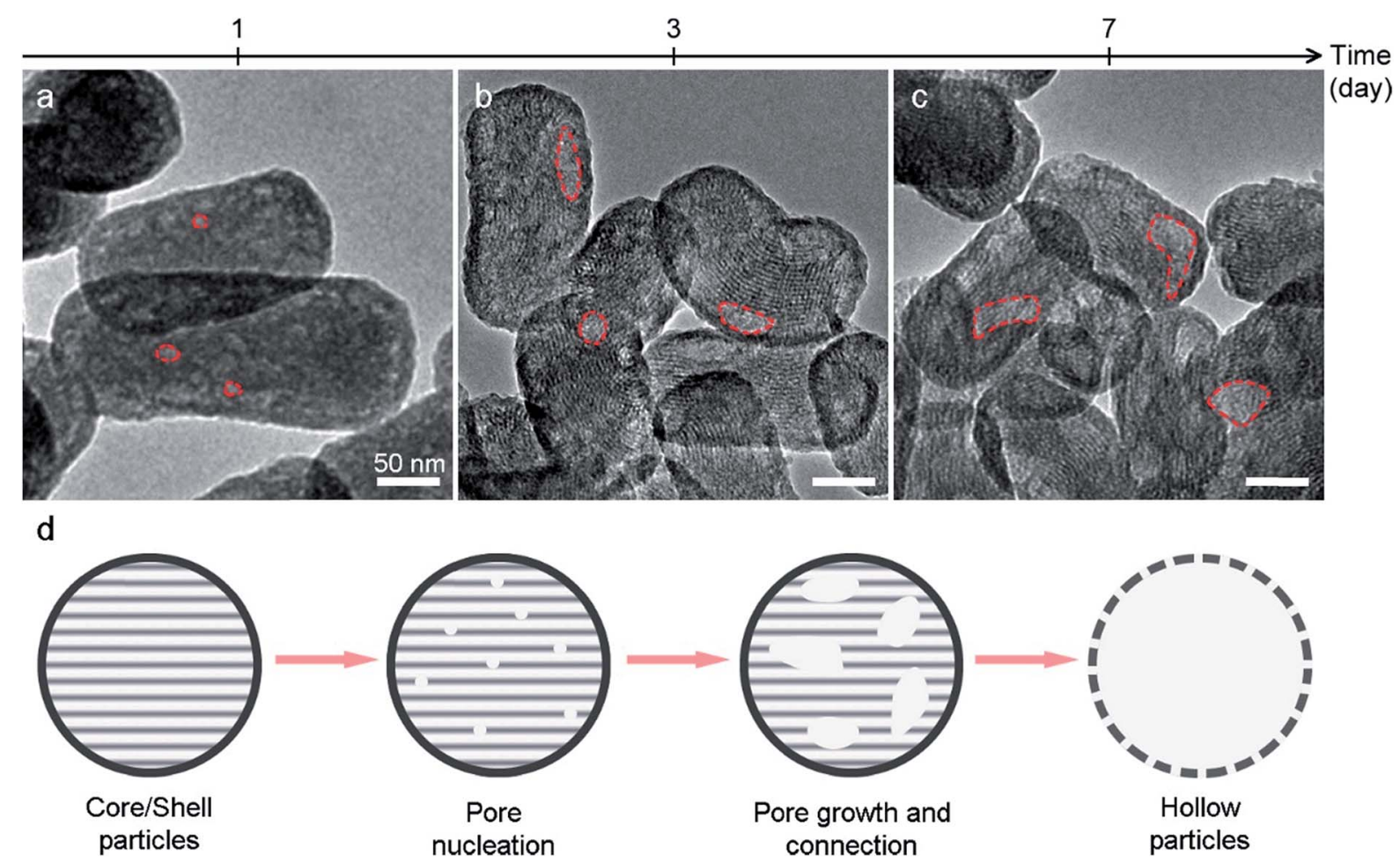

Fig. 8 Mechanism of the core dissolution process. (a-c) TEM images taken at different time intervals during the hollowing of rod-shaped MSNs at room temperature. (a) 1 day, (b) 3 days and (c) 7 days. After 1 day, many small pores around 10 nm were observed. The size of these pores increased with time. Some dissolved areas are outlined in red in all TEM images. (d) Schematic representation of the core dissolution process. 


\section{Conclusions}

In summary, a facile porosity difference based selective silica dissolution strategy to prepare hollow MSNs is reported. First, it was demonstrated that in the presence of the $\mathrm{RB}$ dye the shape of the MSNs was tailored from spheres to rods with controlled aspect ratio. Then, in the presence of excess silica monomers (TEOS) growth of uniform and dense silica shells around MSNs was demonstrated. In PBS, mesoporous cores of these coreshell nanoparticles selectively dissolve due to their higher surface area and less condensed nature compared to the dense shell. After the hollowing process, the shells of the nanoparticles contain mesopores on their surfaces which are nonordered and broad in size distribution which was proved using $\mathrm{N}_{2}$ adsorption-desorption and XRD techniques. The possible selective dissolution mechanism was investigated and it was concluded that dissolution of porous core starts from multiple nucleation sites, these small dissolved sites become larger over time and interconnected with each other until complete core dissolution. We envisage that the porosity difference based selective dissolution route may be applied to synthesize hollow nanoparticles from a wide range of materials with tailored morphologies for many applications including, catalysis, batteries, bioimaging, drug and gene delivery, and optical coatings.

\section{Acknowledgements}

We thank Fahri Emre Öztürk for fruitful discussions. This work is supported by TÜBİTAK under Project no. 111T696. A.Y. is supported by a TÜBITAK-BIDEB Ph.D. fellowship. M.B. acknowledges partial support from the Turkish Academy of Sciences (TÜBA).

\section{Notes and references}

$1 \mathrm{~J} . \mathrm{Hu}, \mathrm{M}$. Chen, X. Fang and L. Wu, Fabrication and application of inorganic hollow spheres, Chem. Soc. Rev., 2011, 40, 5472-5491.

2 X. W. Lou, L. A. Archer and Z. C. Yang, Hollow micro-/ nanostructures: synthesis and applications, Adv. Mater., 2008, 20, 3987-4019.

3 J. Liu, F. Liu, K. Gao, J. S. Wu and D. F. Xue, Recent developments in the chemical synthesis of inorganic porous capsules, J. Mater. Chem., 2009, 19, 6073-6084.

$4 \mathrm{M}$. Chen, L. Wu, S. Zhou and B. You, A method for the fabrication of monodisperse hollow silica spheres, Adv. Mater., 2006, 18, 801-806.

5 Y. Zhao, L.-N. Lin, Y. Lu, S.-F. Chen, L. Dong and S.-H. Yu, Templating synthesis of preloaded doxorubicin in hollow mesoporous silica nanospheres for biomedical applications, Adv. Mater., 2010, 22, 5255-5259.

6 F. Caruso, R. A. Caruso and H. Möhwald, Nanoengineering of inorganic and hybrid hollow spheres by colloidal templating, Science, 1998, 282, 1111-1114.

7 S. O. Obare, N. R. Jana and C. J. Murphy, Preparation of polystyrene- and silica-coated gold nanorods and their use as templates for the synthesis of hollow nanotubes, Nano Lett., 2001, 1, 601-603.

8 Y. Yamada, M. Mizutani, T. Nakamura and K. Yano, Mesoporous microcapsules with decorated inner surface: fabrication and photocatalytic activity, Chem. Mater., 2010, 22, 1695-1703.

9 I. Tissot, J. P. Reymond, F. Lefebvre and E. Bourgeat-Lami, $\mathrm{SiOH}$-functionalized polystyrene latexes. A step toward the synthesis of hollow silica nanoparticles, Chem. Mater., 2002, 14, 1325-1331.

10 W.-J. Zhang, C.-Y. Hong and C.-Y. Pan, Fabrication and characterization of silica nanotubes with controlled dimensions, J. Mater. Chem. A, 2014, 2, 7819-7828.

11 W. R. Zhao, M. D. Lang, Y. S. Li, L. Li and J. L. Shi, Fabrication of uniform hollow mesoporous silica spheres and ellipsoids of tunable size through a facile hardtemplating route, J. Mater. Chem., 2009, 19, 2778-2783.

12 J. Wang, Q. Xiao, H. Zhou, P. Sun, Z. Yuan, B. Li, D. Ding, A.-C. Shi and T. Chen, Budded, mesoporous silica hollow spheres: hierarchical structure controlled by kinetic selfassembly, Adv. Mater., 2006, 18, 3284-3288.

13 M. Mandal and M. Kruk, Family of single-micelle-templated organosilica hollow nanospheres and nanotubes synthesized through adjustment of organosilica/surfactant ratio, Chem. Mater., 2012, 24, 123-132.

14 J. Li, J. Liu, D. Wang, R. Guo, X. Li and W. Qi, Interfacially controlled synthesis of hollow mesoporous silica spheres with radially oriented pore structures, Langmuir, 2010, 26, 12267-12272.

15 H. Djojoputro, X. F. Zhou, S. Z. Qiao, L. Z. Wang, C. Z. Yu and G. Q. Lu, Periodic mesoporous organosilica hollow spheres with tunable wall thickness, J. Am. Chem. Soc., 2006, 128, 6320-6321.

16 K. A. Dahlberg and J. W. Schwank, Synthesis of $\mathrm{Ni} @ \mathrm{SiO}_{2}$ nano-tube particles in a water-in-oil microemulsion template, Chem. Mater., 2012, 24, 2635-2644.

17 Y. Zhao, J. Zhang, W. Li, C. Zhang and B. Han, Synthesis of uniform hollow silica spheres with ordered mesoporous

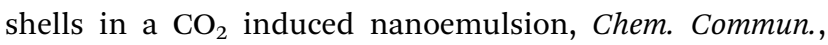
2009, 2365-2367.

18 Q. Sun, P. C. M. M. Magusin, B. Mezari, P. Panine, R. A. van Santen and N. A. J. M. Sommerdijk, The formation of gigantic hollow silica spheres from an $\mathrm{EO}_{76}-\mathrm{PO}_{29}-\mathrm{EO}_{76} /$ butanol/ethanol $/ \mathrm{H}_{2} \mathrm{O}$ quaternary system, J. Mater. Chem., 2005, 15, 256-259.

19 S.-H. Wu, Y. Hung and C.-Y. Mou, Compartmentalized hollow silica nanospheres templated from nanoemulsions, Chem. Mater., 2013, 25, 352-364.

20 Q. Zhang, T. Zhang, J. Ge and Y. Yin, Permeable silica shell through surface-protected etching, Nano Lett., 2008, 8, 28672871.

21 N. Ren, B. Wang, Y.-H. Yang, Y.-H. Zhang, W.-L. Yang, Y.-H. Yue, Z. Gao and Y. Tang, General method for the fabrication of hollow microcapsules with adjustable shell compositions, Chem. Mater., 2005, 17, 2582-2587.

22 M. S. Islam, W. S. Choi and H.-J. Lee, Controlled etching of internal and external structures of $\mathrm{SiO}_{2}$ nanoparticles 
using hydrogen bond of polyelectrolytes, ACS Appl. Mater. Interfaces, 2014, 6, 9563-9571.

23 Y. Chen, H. Chen, L. Guo, Q. He, F. Chen, J. Zhou, J. Feng and J. L. Shi, Hollow/rattle-type mesoporous nanostructures by a structural difference-based selective etching strategy, ACS Nano, 2010, 4, 529-539.

24 X. Fang, C. Chen, Z. Liu, P. Liu and N. Zheng, A cationic surfactant assisted selective etching strategy to hollow mesoporous silica spheres, Nanoscale, 2011, 3, 1632-1639.

25 D. Chen, L. L. Li, F. Q. Tang and S. Qi, Facile and scalable synthesis of tailored silica "nanorattle" structures, Adv. Mater., 2009, 21, 3804-3807.

26 Y. Chen, C. Chu, Y. Zhou, Y. Ru, H. Chen, F. Chen, Q. He, Y. Zhang, L. Zhang and J. L. Shi, Reversible pore-structure evolution in hollow silica nanocapsules: Large pores for siRNA delivery and nanoparticle collecting, Small, 2011, 7, 2935-2944.

27 Y. Chen, H.-R. Chen and J. L. Shi, Construction of homogenous/heterogeneous hollow mesoporous silica nanostructures by silica-etching chemistry: principles, synthesis, and applications, Acc. Chem. Res., 2014, 47, 125137.

28 Y. Chen, Q. Meng, M. Wu, S. Wang, P. Xu, H. Chen, Y. Li, L. Zhang, L. Wang and J. L. Shi, Hollow mesoporous organosilica nanoparticles: a generic intelligent framework-hybridization approach for biomedicine, J. Am. Chem. Soc., 2014, 136, 16326-16334.

29 S.-J. Park, Y.-J. Kim and S.-J. Park, Size-dependent shape evolution of silica nanoparticles into hollow structures, Langmuir, 2008, 24, 12134-12137.

30 Z. Teng, X. Su, Y. Zheng, J. Sun, G. Chen, C. Tian, J. Wang, H. Li, Y. Zhao and G. Lu, Mesoporous silica hollow spheres with ordered radial mesochannels by a spontaneous self-transformation approach, Chem. Mater., 2013, 25, 98-105.

31 Z. Teng, S. Wang, X. Su, G. Chen, Y. Liu, Z. Luo, W. Luo, Y. Tang, H. Ju, D. Zhao and G. Lu, Facile synthesis of yolkshell structured inorganic-organic hybrid spheres with ordered radial mesochannels, Adv. Mater., 2014, 26, 37413747.

32 Y. J. Wong, L. Zhu, W. S. Teo, Y. W. Tan, Y. Yang, C. Wang and $\mathrm{H}$. Chen, Revisiting the Stöber method: inhomogeneity in silica shells, J. Am. Chem. Soc., 2011, 133, 11422-11425.

33 Q. Y. Yu, P. P. Wang, S. Hu, J. F. Hui, J. Zhuang and X. Wang, Hydrothermal synthesis of hollow silica spheres under acidic conditions, Langmuir, 2011, 27, 7185-7191.

34 Y. Chen, H. Chen, D. Zeng, Y. Tian, F. Chen, J. Feng and J. L. Shi, Core-shell structured hollow mesoporous nanocapsules: a potential platform for simultaneous cell imaging and anticancer drug delivery, ACS Nano, 2010, 4, 6001-6013.

35 X. Huang, X. Teng, D. Chen, F. Tang and J. He, The effect of the shape of mesoporous silica nanoparticles on cellular uptake and cell function, Biomaterials, 2010, 31, 438-448.

36 Q. Cai, Z.-S. Luo, W.-Q. Pang, Y.-W. Fan, X.-H. Chen and F.-Z. Cui, Dilute solution routes to various controllable morphologies of MCM-41 silica with a basic medium, Chem. Mater., 2001, 13, 258-263.

37 S. Febvay, D. M. Marini, A. M. Belcher and D. E. Clapham, Targeted cytosolic delivery of cell-impermeable compounds by nanoparticle-mediated, light-triggered endosome disruption, Nano Lett., 2010, 10, 2211-2219.

38 A. Yildirim, G. B. Demirel, R. Erdem, B. Senturk, T. Tekinay and M. Bayindir, Pluronic polymer capped biocompatible mesoporous silica nanocarriers, Chem. Commun., 2013, 49, 9782-9784.

39 S.-H. Wu, C.-Y. Mou and H.-P. Lin, Synthesis of mesoporous silica nanoparticles, Chem. Soc. Rev., 2013, 42, 3862-3875.

40 F. Lu, S.-H. Wu, Y. Hung and C.-Y. Mou, Size effect on cell uptake in well-suspended, uniform mesoporous silica nanoparticles, Small, 2009, 5, 1408-1413.

41 N.-C. Lai, C.-J. Lin, W.-C. Huang and C.-M. Yang, "pH-jump" synthesis of 2D-rectangular $c 2 \mathrm{~mm}$ mesoporous silica materials with helical morphology and extensive void defects, Microporous Mesoporous Mater., 2014, 190, 67-73.

$42 \mathrm{X}$. Du and J. He, Regulation role of ibuprofen toward the morphology of porous silica nanospheres during its in situ encapsulation, J. Colloid Interface Sci., 2010, 345, 269-277.

43 N. Primeau, C. Vautey and M. Langlet, The effect of thermal annealing on aerosol-gel deposited $\mathrm{SiO}_{2}$ films: a FTIR deconvolution study, Thin Solid Films, 1997, 310, 47-56.

44 T. Zhang, J. Ge, Y. Hu, Q. Zhang, S. Aloni and Y. Yin, Formation of hollow silica colloids through a spontaneous dissolution-regrowth process, Angew. Chem., Int. Ed., 2008, 47, 5806-5811.

45 H. Zhu, Y. Ma, Y. Fan and J. Shen, Fourier transform infrared spectroscopy and oxygen luminescence probing combined study of modified sol-gel derived film, Thin Solid Films, 2001, 397, 95-101.

46 R. Filipovic, Z. Obrenovic, I. Stijepovic, L. M. Nikolic and V. V. Srdic, Synthesis of mesoporous silica particles with controlled pore structure, Ceram. Int., 2009, 35, 3347-3353.

$47 \mathrm{~K}$. Kato and Y. Kitano, Solubility and dissolution rate of amorphous silica in distilled and sea water at $20{ }^{\circ} \mathrm{C}, J$. Oceanogr. Soc. Jpn., 1968, 24, 147-152.

48 L. T. Zhuravlev, Concentration of hydroxyl groups on the surface of amorphous silicas, Langmuir, 1987, 3, 316-318. 